\title{
Structural Damage Diagnosis and Prediction Using Machine Learning and Deep Learning Models: Comprehensive Review of Advances
}

\author{
Amir Mosavi \\ School of Built the Environment, Oxford Brookes University, Oxford OX30BP, UK \\ a.mosavi@brookes.ac.uk
}

\begin{abstract}
The loss of integrity and adverse effect on mechanical properties can be concluded as attributing miro/macro-mechanics damage in structures especially in composite structures. Damage as a progressive degradation of material continuity in engineering predictions for any aspects of initiation and propagation, requires to be identified by a trustworthy mechanism to guarantee the safety of structures. Beside the materials design, the structural integrity and health are usually prone to be monitored clearly. One of the most powerful method for detection of damage is machine learning (ML). This paper presents the state of the art of ML methods and their applications in structural damage and prediction. Popular ML methods are identified and the performance and future trends are discussed.
\end{abstract}

Keywords: damage detection; machine learning; principal component analysis; composites; micromechanics of damage; continuum damage mechanics

\section{Acronyms}

ANN Artificial neural network

ELM Extreme learning machine

ML Machine learning

SVM Support vector machine

WNN Wavelet neural networks

DL Deep learning

ARIMA Autoregressive integrated moving average

FFNN Feed-forward neural networks 
MLP Multi layered perceptron

DT Decision tree

RSM Response surface methodology

BPNN Back propagation neural network

CM Centroid mean

ANFIS Adaptive neuro fuzzy inference system

ANP Analytic network process

RF Random forest

NRTL Non-random two-liquid

RNN Recurrent neural network

PLS Partial least squares

DA Discriminant analysis

PCA Principal component analysis

LDA Linear discriminant analysis

SVR Support vector regression

LS Least-squares

SB Sparse Bayesian

MCDM Multi criteria decision making

GP Genetic programming

MLR Multi linear regression

SWARA Step-wise Weight Assessment Ratio Analysis

MOORA Multi Objective Optimization by Ratio Analysis

FFNN Feed-forward neural networks

MLP Multi layered perceptron

DT Decision tree

RSM Response surface methodology

BPNN Back propagation neural network

CM Centroid mean

ANFIS Adaptive neuro fuzzy inference system

ANP Analytic network process

RF Random forest

NRTL Non-random two-liquid

RNN Recurrent neural network

PLS Partial least squares

DA Discriminant analysis 
PCA Principal component analysis

LDA Linear discriminant analysis

SVR Support vector regression

LS Least-squares

SB Sparse Bayesian

MCDM Multi criteria decision making

GP Genetic programming

MLR Multi linear regression

SWARA Step-wise Weight Assessment Ratio Analysis

MOORA Multi Objective Optimization by Ratio Analysis

\section{Introduction}

Structural damage diagnosis and prediction are of utmost importance in various scientific and engineering applications (Cha, Choi, Suh, Mahmoudkhani, \& Büyüköztürk, 2018; Chen et al., 2019; Finotti, Cury, \& Barbosa, 2019). Second paragraph on general damage detection methods and drawback to those methods (Hong et al., 2019; Huang \& Wang, 2018; Jang, Lee, Park, \& Baek, 2018; Kan et al., 2017; Krummenacher, Ong, Koller, Kobayashi, \& Buhmann, 2018; Wang, Hu, \& Zhai, 2018; Z. Zhang et al., 2019). Third paragraph on machine learning suitability and importance of machine learning and deep learning methods on this application (H. Li et al., 2019; Y. Z. Lin, Nie, \& Ma, 2017; F. Ni, Zhang, \& Chen, 2019; Patala, 2019; Pu, Apel, Liu, \& Mitri, 2019; Quaranta et al., 2019). Fourth paragraph of this paper's contribution and the need for a comprehensive review. (Gordan, Razak, Ismail, \& Ghaedi, 2017, 2018) reviews the general application of artificial intelligence methods including soft computing, data mining, optimization methods etc. However, there is a gap in research for a focused and comprehensive review on machine learning and deep learning models (S. Ren, Chen, Li, Chen, \& Li, 2018; Salehi, Das, Biswas, \& Burgueño, 2019).

There has been an enormous evolution in system modeling and intelligence after introducing the early models for deep learning. Deep learning methods very fast emerged and expanded applications in various scientific and engineering domains. Health informatics, energy, urban informatics, safety, security, hydrological systems modeling, economic, bioinformatics, and computational mechanics have been among the early application domains of deep learning. State of the art surveys on the data driven methods 
and machine learning algorithms, indicates that deep learning, along with the ensemble and hybrid machine learning methods are the future of data science. Further comparative studies report that deep learning models and hybrid machine learning models often outperform conventional machine learning models. Figure 1 represents the rapid rise in the applications of various deep learning methods during the past five years. Deep learning methods are fast evolving for higher performance. Literature includes adequate review papers on the progressing algorithms in particular application domains, e.g., renewable energy forecasting, cardiovascular image analysis, resolution imaging, radiology, 3D sensed data classification, 3D sensed data classification, multimedia analytics, sentiment classification, text detection, transportation systems, activity recognition in radar, hyperspectral, medical ultrasound analysis, image cytometry, and apache spark. However, a simplified list of deep learning methods has not been communicated so far. Thus, there is a gap in research in introducing the deep learning methods and summarize the methods and application in a brief, yet communicative paper. Consequently, this paper aims at providing a comprehensive list of the most popular deep learning methods and their notable applications. In every section, one deep learning method is introduced, and the notable applications related to that method are listed. The description of each deep learning method and the function of each building block is explained.

\section{Survey methodology}

The primary goal of this literature survey is to present the state of the art of ML models in the individual application areas of structural damage diagnosis. Accordingly, the research methodology has been developed to identify, classify and review the notable peer-reviewed articles in design and implementation of sustainable business models in top-level subject fields. The Web-of-Science and elsevier scopus are used for the implementation of the search queries of "defect or damage or crack" and "ml method ${ }_{1-\mathrm{n}}$ " for title, abstract and keywords the relevant literatures are identified. the query of (title-abs-key (defect or damage or crack) and title-abs-key $\left.\left(\operatorname{ml~method~}_{1-n}\right)\right)$ in addition to the query of (title-abskey (defect or damage or crack) and title-abs-key (dl method $\left.{ }_{1-n}\right)$ ) would result in 21,933 documents. however, through auxiliary search keywords such as "mechanic* and

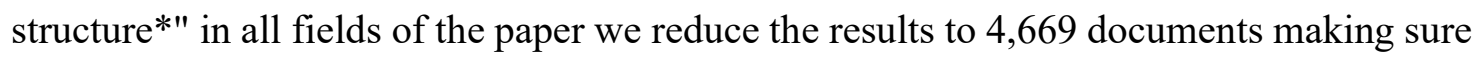
that the most relevant papers are identified, which forms our initial database. Reading in detail the articles' relevancy downed the numbers to 150 articles for the final consideration. 
The research methodology follows a comprehensive and structured workflow based on a systematic database search and cross-reference snowballing. The flowchart of the research methodology is presented in figure 1. The method is considered as a modified version of review proposed by Easterby-Smith et al. (2015). In the first step the search queries explore the Thomson Reuters Web-of-Science and Elsevier Scopus databases. In the second step the abstract and keywords of the identified articles are browsed to identify the relevant literature and exclude the irrelevant ones. In step three the database of the relevant articles is created. In step four, the article is carefully read, and the category of the application is identified accordingly. In this step the expert-based knowledge and the initial preferences would influence the number and the type of the categories. In step five we decide on generating a new category and export the article in a new table of application domain or pass the article to step six where a category would host an article in its table. Once a category is created for a new article, in step seven, we pass that article to that category. In step eight we save the content of our database in various categories, update the content of the tables, and review the papers. This workflow will be repeated until sorting out all the papers.

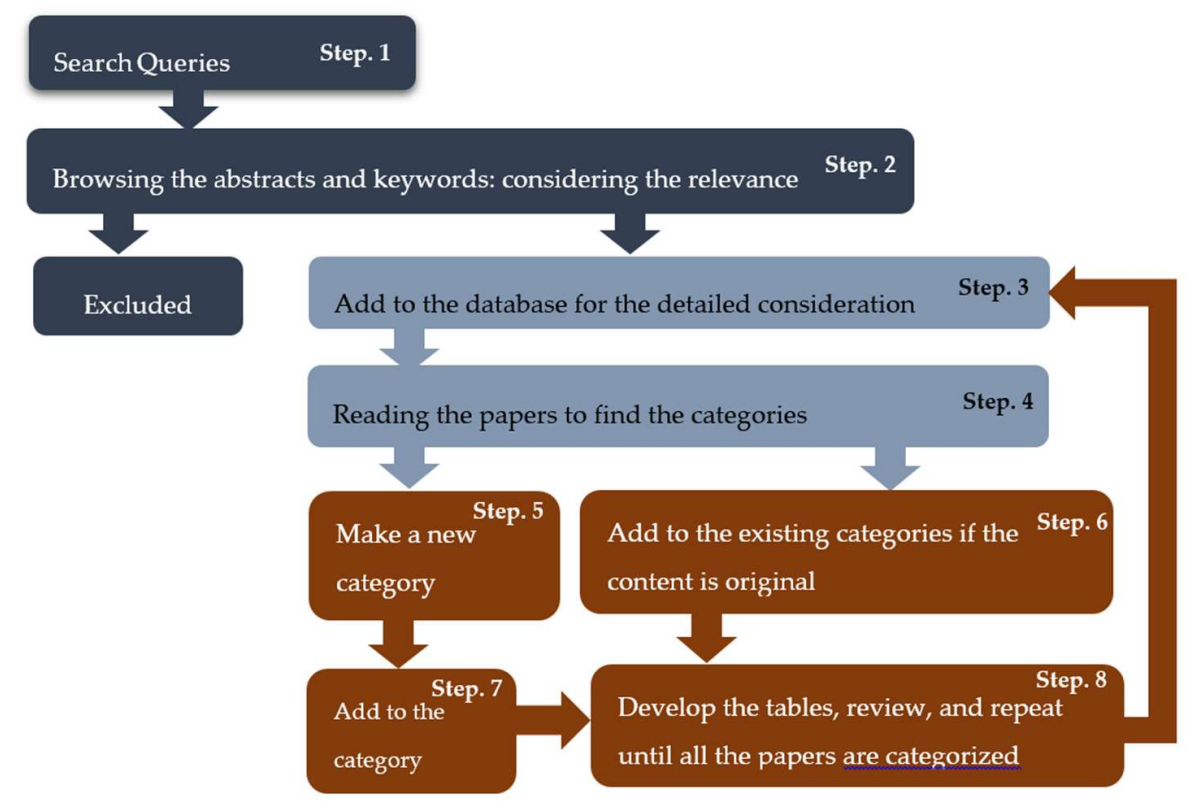

Figure 1. Flowchart of the methodology of research 


\section{Machine learning methods}

The survey methodology classifies the machine learning methods in seven groups, i.e. ANN-based, SVM-based, Tree-based models, Ensembles, Bayesians, Logistic regressions, and Neuro-Fuzzy. The last group is deep learning which has been considered separately. The notable papers have been reviewed in individual classes.

\subsection{ANN}

ANN can prepare general frameworks for analyzing damaged induced materials. Due to the fact that artificial neural networks have various applications such as accurate prediction of complex material behavior, it could be applied for damage detection and structural integrities in corresponding multiple-variable problems.

Table 1. Notable ANN-based models for structural damage diagnosis and prediction

\begin{tabular}{|c|c|c|c|}
\hline Reference & Year & Contribution & Application \\
\hline $\begin{array}{l}\text { Zhang Z., Hong } \\
\text { Y., Hou B., } \\
\text { Zhang Z., } \\
\text { Negahban M., } \\
\text { Zhang J. }\end{array}$ & 2019 & $\begin{array}{l}\text { Accelerated discoveries } \\
\text { of mechanical properties } \\
\text { of graphene using } \\
\text { machine learning and } \\
\text { high-throughput } \\
\text { computation }\end{array}$ & \\
\hline $\begin{array}{l}\text { Jafari-Marandi } \\
\text { R., Khanzadeh } \\
\text { M., Tian W., } \\
\text { Smith B., Bian } \\
\text { L. }\end{array}$ & 2019 & $\begin{array}{l}\text { From in-situ monitoring } \\
\text { toward high-throughput } \\
\text { process control: cost- } \\
\text { driven decision-making } \\
\text { framework for laser- } \\
\text { based additive } \\
\text { manufacturing }\end{array}$ & $\begin{array}{l}\text { Additive manufacturing (AM); } \\
\text { Artificial neural networks (ANN); } \\
\text { Porosity prediction; Self-organizing } \\
\text { error-drive neural networks } \\
\text { (SOEDNN); Thermal history }\end{array}$ \\
\hline $\begin{array}{l}\text { Gomes G.F., de } \\
\text { Almeida F.A., } \\
\text { Junqueira D.M., } \\
\text { da Cunha S.S., } \\
\text { Jr., Ancelotti } \\
\text { A.C., Jr. }\end{array}$ & 2019 & $\begin{array}{l}\text { Optimized damage } \\
\text { identification in CFRP } \\
\text { plates by reduced mode } \\
\text { shapes and GA-ANN } \\
\text { methods }\end{array}$ & $\begin{array}{l}\text { Artificial neural networks; Composite } \\
\text { plates; Damage identification; Inverse } \\
\text { problem; Sensor placement } \\
\text { optimization; Structural health } \\
\text { monitoring }\end{array}$ \\
\hline $\begin{array}{l}\text { Finotti R.P., } \\
\text { Cury A.A., } \\
\text { Barbosa F.S. }\end{array}$ & 2019 & $\begin{array}{l}\text { An SHM approach using } \\
\text { machine learning and } \\
\text { statistical indicators } \\
\text { extracted from raw } \\
\text { dynamic measurements }\end{array}$ & $\begin{array}{l}\text { Computational intelligence; Damage } \\
\text { identification; Dynamic } \\
\text { measurement; Structural dynamic; } \\
\text { Structural health monitoring; } \\
\text { Vibration monitoring }\end{array}$ \\
\hline
\end{tabular}




\begin{tabular}{|c|c|c|c|}
\hline $\begin{array}{l}\text { Pu Y., Apel } \\
\text { D.B., Liu V., } \\
\text { Mitri H. }\end{array}$ & 2019 & $\begin{array}{l}\text { Machine learning } \\
\text { methods for rockburst } \\
\text { prediction-state-of-the-art } \\
\text { review }\end{array}$ & $\begin{array}{l}\text { Artificial neural network; Burst } \\
\text { liability; Deep learning; Rockburst } \\
\text { prediction; Support vector machine }\end{array}$ \\
\hline $\begin{array}{l}\text { Wong E.W.C., } \\
\text { Kim D.K. }\end{array}$ & 2018 & $\begin{array}{l}\text { A simplified method to } \\
\text { predict fatigue damage of } \\
\text { TTR subjected to short- } \\
\text { term VIV using artificial } \\
\text { neural network }\end{array}$ & $\begin{array}{l}\text { Artificial neural network; Current; } \\
\text { Fatigue damage; Riser; Top- } \\
\text { tensioned riser; Vortex-induced } \\
\text { vibration }\end{array}$ \\
\hline $\begin{array}{l}\text { Nie W., Zhao } \\
\text { Z.Y., Goh } \\
\text { A.T.C., Song } \\
\text { M.K., Guo W., } \\
\text { Zhu X. }\end{array}$ & 2018 & $\begin{array}{l}\text { Performance based } \\
\text { support design for } \\
\text { horseshoe-shaped rock } \\
\text { caverns using 2D } \\
\text { numerical analysis }\end{array}$ & $\begin{array}{l}\text { Artificial neural network; } \\
\text { Convergence confinement method; } \\
\text { Rock cavern; Support design }\end{array}$ \\
\hline Gedik N. & 2018 & $\begin{array}{l}\text { Least squares support } \\
\text { vector mechanics to } \\
\text { predict the stability } \\
\text { number of rubble-mound } \\
\text { breakwaters }\end{array}$ & $\begin{array}{l}\text { Least squares support vector } \\
\text { mechanics; Particle swarm } \\
\text { optimization; Rubble-mound } \\
\text { breakwater; Stability number }\end{array}$ \\
\hline $\begin{array}{l}\text { Rezaniaiee } \\
\text { Aqdam H., } \\
\text { Ettefagh M.M., } \\
\text { Hassannejad R. }\end{array}$ & 2018 & $\begin{array}{l}\text { Health monitoring of } \\
\text { mooring lines in floating } \\
\text { structures using artificial } \\
\text { neural networks }\end{array}$ & $\begin{array}{l}\text { Damage diagnosis; Finite element } \\
\text { method; Mooring lines; Radial basis } \\
\text { neural networks; Structural health } \\
\text { monitoring; Uncertainty }\end{array}$ \\
\hline $\begin{array}{l}\text { Cha Y.-J., Choi } \\
\text { W., Suh G., } \\
\text { Mahmoudkhani } \\
\text { S., Büyüköztürk } \\
\text { O. }\end{array}$ & 2018 & $\begin{array}{l}\text { Autonomous Structural } \\
\text { Visual Inspection Using } \\
\text { Region-Based Deep } \\
\text { Learning for Detecting } \\
\text { Multiple Damage Types }\end{array}$ & \\
\hline $\begin{array}{l}\text { Wang Z., Hu } \\
\text { M., Zhai G. }\end{array}$ & 2018 & $\begin{array}{l}\text { Application of deep } \\
\text { learning architectures for } \\
\text { accurate and rapid } \\
\text { detection of internal } \\
\text { mechanical damage of } \\
\text { blueberry using } \\
\text { hyperspectral } \\
\text { transmittance data }\end{array}$ & $\begin{array}{l}\text { Convolutional neural networks; Fruit } \\
\text { quality detection; Hyperspectral } \\
\text { transmittance image; Internal } \\
\text { mechanical damage detection; } \\
\text { Machine learning }\end{array}$ \\
\hline $\begin{array}{l}\text { Krummenacher } \\
\text { G., Ong C.S., } \\
\text { Koller S., } \\
\text { Kobayashi S., } \\
\text { Buhmann J.M. }\end{array}$ & 2018 & $\begin{array}{l}\text { Wheel Defect Detection } \\
\text { with Machine Learning }\end{array}$ & $\begin{array}{l}\text { artificial neural networks; Machine } \\
\text { learning; pattern analysis; railway } \\
\text { accidents; railway safety; statistical } \\
\text { learning; supervised learning; support } \\
\text { vector machines; wavelet transforms }\end{array}$ \\
\hline $\begin{array}{l}\text { AminShokravi } \\
\text { A., Eskandar H., } \\
\text { Derakhsh A.M., } \\
\text { Rad H.N., } \\
\text { Ghanadi A. }\end{array}$ & 2018 & $\begin{array}{l}\text { The potential application } \\
\text { of particle swarm } \\
\text { optimization algorithm } \\
\text { for forecasting the air- } \\
\text { overpressure induced by } \\
\text { mine blasting }\end{array}$ & ANN; AOp; Blasting; PSO \\
\hline
\end{tabular}




\begin{tabular}{|c|c|c|c|}
\hline $\begin{array}{l}\text { Gordan M., } \\
\text { Razak H.A., } \\
\text { Ismail Z., } \\
\text { Ghaedi K. }\end{array}$ & 2018 & $\begin{array}{l}\text { Data mining based } \\
\text { damage identification } \\
\text { using imperialist } \\
\text { competitive algorithm } \\
\text { and artificial neural } \\
\text { network }\end{array}$ & $\begin{array}{l}\text { Artificial neural network; Damage } \\
\text { detection; Data mining; Hybrid } \\
\text { algorithm; Imperial competitive } \\
\text { algorithm; Structural health } \\
\text { monitoring }\end{array}$ \\
\hline $\begin{array}{l}\text { Ghritlahre H.K., } \\
\text { Prasad R.K. }\end{array}$ & 2018 & $\begin{array}{l}\text { Exergetic performance } \\
\text { prediction of a roughened } \\
\text { solar air heater using } \\
\text { artificial neural network }\end{array}$ & $\begin{array}{l}\text { Artificial neural network; Exergy } \\
\text { analysis; Learning algorithm; Multi- } \\
\text { layer perceptron; Solar air heater }\end{array}$ \\
\hline $\begin{array}{l}\text { Rojas-Moraleda } \\
\text { R., Valous } \\
\text { N.A., Gowen } \\
\text { A., Esquerre C., } \\
\text { Härtel S., } \\
\text { Salinas L., } \\
\text { O'Donnell C. }\end{array}$ & 2017 & $\begin{array}{l}\text { A frame-based ANN for } \\
\text { classification of } \\
\text { hyperspectral images: } \\
\text { assessment of mechanical } \\
\text { damage in mushrooms }\end{array}$ & $\begin{array}{l}\text { Artificial neural networks; Frame- } \\
\text { based classification; Imaging } \\
\text { spectroscopy; Mechanical damage; } \\
\text { Salient point detectors }\end{array}$ \\
\hline $\begin{array}{l}\text { Lin Y.-Z., Nie } \\
\text { Z.-H., Ma H.- } \\
\text { W. }\end{array}$ & 2017 & \begin{tabular}{l|} 
Structural Damage \\
Detection with \\
Automatic Feature- \\
Extraction through Deep \\
Learning
\end{tabular} & \\
\hline $\begin{array}{l}\text { Çalık A., } \\
\text { Yildırım S., } \\
\text { Tosun E. }\end{array}$ & 2017 & $\begin{array}{l}\text { Estimation of crack } \\
\text { propagation in polymer } \\
\text { electrolyte membrane } \\
\text { fuel cell under vibration } \\
\text { conditions }\end{array}$ & $\begin{array}{l}\text { Artificial neural network; Crack } \\
\text { propagation; Mechanical vibration; } \\
\text { Polymer electrolyte membrane fuel } \\
\text { cell }\end{array}$ \\
\hline $\begin{array}{l}\text { Tan Z.X., } \\
\text { Thambiratnam } \\
\text { D.P., Chan } \\
\text { T.H.T., Abdul } \\
\text { Razak H. }\end{array}$ & 2017 & $\begin{array}{l}\text { Detecting damage in } \\
\text { steel beams using modal } \\
\text { strain energy based } \\
\text { damage index and } \\
\text { Artificial Neural } \\
\text { Network }\end{array}$ & $\begin{array}{l}\text { Artificial Neural Network; Damage } \\
\text { index; Damage location; Damage } \\
\text { prediction; Damage scenarios; } \\
\text { Damage severity; Failure prevention; } \\
\text { Modal strain energy; Vibration based } \\
\text { technique }\end{array}$ \\
\hline $\begin{array}{l}\text { Samareh H., } \\
\text { Khoshrou S.H., } \\
\text { Shahriar K., } \\
\text { Ebadzadeh } \\
\text { M.M., Eslami } \\
\text { M. }\end{array}$ & 2017 & $\begin{array}{l}\text { Optimization of a } \\
\text { nonlinear model for } \\
\text { predicting the ground } \\
\text { vibration using the } \\
\text { combinational particle } \\
\text { swarm optimization- } \\
\text { genetic algorithm }\end{array}$ & $\begin{array}{l}\text { Artificial neural network; Blasting; } \\
\text { Genetic algorithm; Geo-mechanics } \\
\text { properties of rock mass; Ground } \\
\text { vibration; Particle swarm } \\
\text { optimization }\end{array}$ \\
\hline $\begin{array}{l}\text { Gordan M., } \\
\text { Razak H.A., } \\
\text { Ismail Z., } \\
\text { Ghaedi K. }\end{array}$ & 2017 & $\begin{array}{l}\text { Recent developments in } \\
\text { damage identification of } \\
\text { structures using data } \\
\text { mining }\end{array}$ & $\begin{array}{l}\text { Artificial neural network; Data } \\
\text { mining technique; Genetic algorithm; } \\
\text { Principal component analysis; } \\
\text { Structural damage detection }\end{array}$ \\
\hline $\begin{array}{l}\text { Choi C.K., Kim } \\
\text { J.S., Yoo H.H. }\end{array}$ & 2016 & $\begin{array}{l}\text { Identification of location } \\
\text { and size of a defect in a } \\
\text { structural system }\end{array}$ & $\begin{array}{l}\text { Active external moment; Artificial } \\
\text { neural network (ANN); Fault }\end{array}$ \\
\hline
\end{tabular}




\begin{tabular}{|c|c|c|c|}
\hline & & $\begin{array}{l}\text { employing active } \\
\text { external excitation and } \\
\text { hybrid feature vector } \\
\text { components in HMM }\end{array}$ & $\begin{array}{l}\text { diagnosis; Hidden Markov model } \\
\text { (HMM); Structural system }\end{array}$ \\
\hline $\begin{array}{l}\text { Bissacot } \\
\text { A.C.G., Salgado } \\
\text { S.A.B., } \\
\text { Balestrassi P.P., } \\
\text { Paiva A.P., } \\
\text { Zambroni Souza } \\
\text { A.C., Wazen R. } \\
\end{array}$ & 2016 & $\begin{array}{l}\text { Comparison of neural } \\
\text { networks and logistic } \\
\text { regression in assessing } \\
\text { the occurrence of failures } \\
\text { in steel structures of } \\
\text { transmission lines }\end{array}$ & $\begin{array}{l}\text { Artificial neural networks; Fall of } \\
\text { metal structures; Logistic regression; } \\
\text { ROC curves; Transmission lines }\end{array}$ \\
\hline $\begin{array}{l}\text { Guruprasad R., } \\
\text { Behera B.K. }\end{array}$ & 2015 & $\begin{array}{l}\text { Comparative Analysis of } \\
\text { Soft Computing Models } \\
\text { in Prediction of Bending } \\
\text { Rigidity of Cotton } \\
\text { Woven Fabrics }\end{array}$ & $\begin{array}{l}\text { ANFIS; ANN; Bending rigidity; } \\
\text { BPNN; GANN }\end{array}$ \\
\hline $\begin{array}{l}\text { Alves V., Cury } \\
\text { A., Roitman N., } \\
\text { Magluta C., } \\
\text { Cremona C. }\end{array}$ & 2015 & $\begin{array}{l}\text { Structural modification } \\
\text { assessment using } \\
\text { supervised learning } \\
\text { methods applied to } \\
\text { vibration data } \\
\end{array}$ & $\begin{array}{l}\text { Damage assessment; Learning } \\
\text { algorithms; Pattern recognition; } \\
\text { SHM; Symbolic data }\end{array}$ \\
\hline $\begin{array}{l}\text { Güneyisi E.M., } \\
\text { Mermerdaş K., } \\
\text { Güneyisi E., } \\
\text { Gesoğlu M. }\end{array}$ & 2015 & $\begin{array}{l}\text { Numerical modeling of } \\
\text { time to corrosion induced } \\
\text { cover cracking in } \\
\text { reinforced concrete using } \\
\text { soft-computing based } \\
\text { methods }\end{array}$ & $\begin{array}{l}\text { Experimental database; Modeling; } \\
\text { Reinforced concrete; Steel } \\
\text { reinforcement corrosion; Time to } \\
\text { cover cracking }\end{array}$ \\
\hline $\begin{array}{l}\text { Harish N., } \\
\text { Mandal S., Rao } \\
\text { S., Patil S.G. }\end{array}$ & 2015 & $\begin{array}{l}\text { Particle Swarm } \\
\text { Optimization based } \\
\text { support vector machine } \\
\text { for damage level } \\
\text { prediction of non- } \\
\text { reshaped berm } \\
\text { breakwater }\end{array}$ & $\begin{array}{l}\text { Berm breakwater; Damage level; } \\
\text { Non-reshaped; PSO-SVM; SVM }\end{array}$ \\
\hline $\begin{array}{l}\text { Meruane V., } \\
\text { Ortiz-Bernardin } \\
\text { A. }\end{array}$ & 2015 & $\begin{array}{l}\text { Structural damage } \\
\text { assessment using linear } \\
\text { approximation with } \\
\text { maximum entropy and } \\
\text { transmissibility data }\end{array}$ & $\begin{array}{l}\text { Linear approximation; Maximum- } \\
\text { entropy principle; Structural damage } \\
\text { assessment; Supervised learning } \\
\text { algorithms }\end{array}$ \\
\hline
\end{tabular}

ANN based models include a great deal of models for damage modeling. (Alves, Cury, Roitman, Magluta, \& Cremona, 2015; AminShokravi, Eskandar, Derakhsh, Rad, \& Ghanadi, 2018; Bissacot et al., 2016; Çalık, Yıldırım, \& Tosun, 2017; Cha et al., 2018; Choi, Kim, \& Yoo, 2016; Finotti et al., 2019; Gedik, 2018; Ghritlahre \& Prasad, 2018; 
Gomes, de Almeida, Junqueira, da Cunha, \& Ancelotti, 2019; Gordan et al., 2017, 2018; Güneyisi, Mermerdaş, Güneyisi, \& Gesoğlu, 2015; Guruprasad \& Behera, 2015; Harish, Mandal, Rao, \& Patil, 2015; Jafari-Marandi, Khanzadeh, Tian, Smith, \& Bian, 2019; Krummenacher et al., 2018; Y. Z. Lin et al., 2017; Meruane \& Ortiz-Bernardin, 2015; Nie et al., 2018; Pu et al., 2019; Rezaniaiee Aqdam, Ettefagh, \& Hassannejad, 2018; RojasMoraleda et al., 2017; Samareh, Khoshrou, Shahriar, Ebadzadeh, \& Eslami, 2017; Tan, Thambiratnam, Chan, \& Abdul Razak, 2017; Wang et al., 2018; Wong \& Kim, 2018; Z. Zhang et al., 2019)

Table. 2 Notable Support vectors (SVM) models

\begin{tabular}{|c|c|c|c|}
\hline Authors & Year & Title & Author Keywords \\
\hline $\begin{array}{l}\text { Nair A., Cai C.S., } \\
\text { Kong X. }\end{array}$ & 2019 & $\begin{array}{l}\text { Studying Failure Modes of GFRP } \\
\text { Laminate Coupons Using AE Pattern- } \\
\text { Recognition Method }\end{array}$ & $\begin{array}{l}\text { Acoustic emission; Failure mode } \\
\text { identification; Glass fiber reinforced } \\
\text { polymer (GFRP) laminate coupon; } \mathrm{k} \text { - } \\
\text { means clustering; Multilayer perceptron; } \\
\text { Pattern recognition; Support vector } \\
\text { machine }\end{array}$ \\
\hline $\begin{array}{l}\text { Zhang Z., Hong } \\
\text { Y., Hou B., Zhang } \\
\text { Z., Negahban M., } \\
\text { Zhang J. }\end{array}$ & 2019 & $\begin{array}{l}\text { Accelerated discoveries of mechanical } \\
\text { properties of graphene using machine } \\
\text { learning and high-throughput } \\
\text { computation }\end{array}$ & \\
\hline $\begin{array}{l}\text { Nair A., Cai C.S., } \\
\text { Kong X. }\end{array}$ & 2019 & $\begin{array}{l}\text { Acoustic emission pattern recognition } \\
\text { in CFRP retrofitted RC beams for } \\
\text { failure mode identification }\end{array}$ & $\begin{array}{l}\text { Acoustic emission; CFRP retrofitted RC } \\
\text { beams; Failure mode identification; K- } \\
\text { means clustering; Multilayer perceptron; } \\
\text { Pattern recognition; Support vector } \\
\text { machine }\end{array}$ \\
\hline $\begin{array}{l}\text { Forero-Ramírez J.- } \\
\text { C., Restrepo-Girón } \\
\text { A.-D., Nope- } \\
\text { Rodríguez S.-E. }\end{array}$ & 2019 & $\begin{array}{l}\text { Detection of Internal Defects in } \\
\text { Carbon Fiber Reinforced Plastic Slabs } \\
\text { Using Background Thermal } \\
\text { Compensation by Filtering and Support } \\
\text { Vector Machines }\end{array}$ & $\begin{array}{l}\text { Background thermal compensation by } \\
\text { filtering (BTCF); Carbon fiber reinforced } \\
\text { plastic (CFRP); Feature selection; } \\
\text { Infrared thermography (IT); Support } \\
\text { vector machines (SVM) }\end{array}$ \\
\hline $\begin{array}{l}\text { Finotti R.P., Cury } \\
\text { A.A., Barbosa F.S. }\end{array}$ & 2019 & $\begin{array}{l}\text { An SHM approach using machine } \\
\text { learning and statistical indicators } \\
\text { extracted from raw dynamic } \\
\text { measurements }\end{array}$ & $\begin{array}{l}\text { Computational intelligence; Damage } \\
\text { identification; Dynamic measurement; } \\
\text { Structural dynamic; Structural health } \\
\text { monitoring; Vibration monitoring }\end{array}$ \\
\hline $\begin{array}{l}\text { Pu Y., Apel D.B., } \\
\text { Liu V., Mitri H. }\end{array}$ & 2019 & $\begin{array}{l}\text { Machine learning methods for } \\
\text { rockburst prediction-state-of-the-art } \\
\text { review }\end{array}$ & $\begin{array}{l}\text { Artificial neural network; Burst liability; } \\
\text { Deep learning; Rockburst prediction; } \\
\text { Support vector machine }\end{array}$ \\
\hline Gedik N. & 2018 & $\begin{array}{l}\text { Least squares support vector } \\
\text { mechanics to predict the stability } \\
\text { number of rubble-mound breakwaters }\end{array}$ & $\begin{array}{l}\text { Least squares support vector mechanics; } \\
\text { Particle swarm optimization; Rubble- } \\
\text { mound breakwater; Stability number }\end{array}$ \\
\hline
\end{tabular}




\begin{tabular}{|c|c|c|c|}
\hline Lin T.-K. & 2018 & $\begin{array}{l}\text { An edge-feature-description-based } \\
\text { scheme combined with support vector } \\
\text { machines for the detection of vortex- } \\
\text { induced vibration }\end{array}$ & $\begin{array}{l}\text { Edge feature description; Hybrid vision- } \\
\text { based method; Support vector machines; } \\
\text { Vortex-induced vibration }\end{array}$ \\
\hline $\begin{array}{l}\text { Krummenacher G., } \\
\text { Ong C.S., Koller } \\
\text { S., Kobayashi S., } \\
\text { Buhmann J.M. }\end{array}$ & 2018 & $\begin{array}{l}\text { Wheel Defect Detection with Machine } \\
\text { Learning }\end{array}$ & $\begin{array}{l}\text { artificial neural networks; Machine } \\
\text { learning; pattern analysis; railway } \\
\text { accidents; railway safety; statistical } \\
\text { learning; supervised learning; support } \\
\text { vector machines; wavelet transforms }\end{array}$ \\
\hline $\begin{array}{l}\text { Rojas-Moraleda } \\
\text { R., Valous N.A., } \\
\text { Gowen A., } \\
\text { Esquerre C., } \\
\text { Härtel S., Salinas } \\
\text { L., O’Donnell C. }\end{array}$ & 2017 & $\begin{array}{l}\text { A frame-based ANN for classification } \\
\text { of hyperspectral images: assessment of } \\
\text { mechanical damage in mushrooms }\end{array}$ & $\begin{array}{l}\text { Artificial neural networks; Frame-based } \\
\text { classification; Imaging spectroscopy; } \\
\text { Mechanical damage; Salient point } \\
\text { detectors }\end{array}$ \\
\hline $\begin{array}{l}\text { Yi Q., Wang H., } \\
\text { Guo R., Li S., } \\
\text { Jiang Y. }\end{array}$ & 2017 & $\begin{array}{l}\text { Laser ultrasonic quantitative } \\
\text { recognition based on wavelet packet } \\
\text { fusion algorithm and SVM }\end{array}$ & $\begin{array}{l}\text { Dimension reduction; Laser ultrasonic; } \\
\text { Quantitative recognition; SVM } \\
\text { classification; Wavelet packet fusion }\end{array}$ \\
\hline $\begin{array}{l}\text { Alves V., Cury A., } \\
\text { Roitman N., } \\
\text { Magluta C., } \\
\text { Cremona C. }\end{array}$ & 2015 & $\begin{array}{l}\text { Structural modification assessment } \\
\text { using supervised learning methods } \\
\text { applied to vibration data }\end{array}$ & $\begin{array}{l}\text { Damage assessment; Learning algorithms; } \\
\text { Pattern recognition; SHM; Symbolic data }\end{array}$ \\
\hline $\begin{array}{l}\text { Lu S., Jiang M., } \\
\text { Sui Q., Sai Y., Jia } \\
\text { L. }\end{array}$ & 2015 & $\begin{array}{l}\text { Damage identification system of CFRP } \\
\text { using fiber bragg grating sensors }\end{array}$ & $\begin{array}{l}\text { CFRP structural damage identification; } \\
\text { Fiber bragg grating; Multi-class C-suppor } \\
\text { vector classification; One-class support } \\
\text { vector machines; Principal component } \\
\text { analysis }\end{array}$ \\
\hline $\begin{array}{l}\text { Harish N., Mandal } \\
\text { S., Rao S., Patil } \\
\text { S.G. }\end{array}$ & 2015 & $\begin{array}{l}\text { Particle Swarm Optimization based } \\
\text { support vector machine for damage } \\
\text { level prediction of non-reshaped berm } \\
\text { breakwater }\end{array}$ & $\begin{array}{l}\text { Berm breakwater; Damage level; Non- } \\
\text { reshaped; PSO-SVM; SVM }\end{array}$ \\
\hline
\end{tabular}

SVM have gained popularity in modeling the damage. (Alves et al., 2015; Finotti et al., 2019; Forero-Ramírez, Restrepo-Girón, \& Nope-Rodríguez, 2019; Gedik, 2018; Harish et al., 2015; Krummenacher et al., 2018; T. K. Lin, 2018; Lu, Jiang, Sui, Sai, \& Jia, 2015; Nair, Cai, \& Kong, 2019a, 2019b; Pu et al., 2019; Rojas-Moraleda et al., 2017; Yi, Wang, Guo, Li, \& Jiang, 2017; Z. Zhang et al., 2019)

\section{Tree-based models;}

Decision trees (DTs), Classification and Regression Trees (CART) 
Table 3. Notable tree-based models

\begin{tabular}{|c|c|c|c|}
\hline Authors & Year & Source title & Author Keywords \\
\hline $\begin{array}{l}\text { Andrejiova M., } \\
\text { Grincova A., } \\
\text { Marasova D. }\end{array}$ & 2019 & $\begin{array}{l}\text { Engineering } \\
\text { Failure } \\
\text { Analysis }\end{array}$ & $\begin{array}{l}\text { Classification model; Damage; } \\
\text { Decision tree; Regression } \\
\text { analysis; Rubber-textile } \\
\text { conveyor belt }\end{array}$ \\
\hline $\begin{array}{l}\text { Dia A., Dieng L., } \\
\text { Gaillet L., Gning P.B. }\end{array}$ & 2019 & Heliyon & $\begin{array}{l}\text { Acoustics; Materials science; } \\
\text { Mechanical engineering }\end{array}$ \\
\hline $\begin{array}{l}\text { Bhowmik B., } \\
\text { Krishnan M., Hazra } \\
\text { B., Pakrashi V. }\end{array}$ & 2019 & $\begin{array}{l}\text { Structural } \\
\text { Health } \\
\text { Monitoring }\end{array}$ & $\begin{array}{l}\text { damage-sensitive features; real- } \\
\text { time damage detection; } \\
\text { Recursive singular spectral } \\
\text { analysis; structural health } \\
\text { monitoring; time-varying } \\
\text { autoregressive modeling }\end{array}$ \\
\hline $\begin{array}{l}\text { Noori Hoshyar A., } \\
\text { Samali B., } \\
\text { Liyanapathirana R., } \\
\text { Taghavipour S. }\end{array}$ & 2019 & $\begin{array}{l}\text { Structural } \\
\text { Health } \\
\text { Monitoring }\end{array}$ & $\begin{array}{l}\text { damage index; de-noising; } \\
\text { mother wavelets; severity } \\
\text { analysis; smart aggregate } \\
\text { sensors; Structural health } \\
\text { monitoring; wavelet de-noising }\end{array}$ \\
\hline $\begin{array}{l}\text { Egnew A.C., Roueche } \\
\text { D.B., Prevatt D.O. }\end{array}$ & 2018 & $\begin{array}{l}\text { Natural Hazards } \\
\text { Review }\end{array}$ & \\
\hline $\begin{array}{l}\text { Pérez-Ruiz M., Rallo } \\
\text { P., Jiménez M.R., } \\
\text { Garrido-Izard M., } \\
\text { Suárez M.P., } \\
\text { Casanova L., Valero } \\
\text { C., Martínez-Guanter } \\
\text { J., Morales-Sillero A. }\end{array}$ & 2018 & $\begin{array}{l}\text { Sensors } \\
\text { (Switzerland) }\end{array}$ & $\begin{array}{l}\text { Canopy volume; Fruit damage; } \\
\text { Laser scanning; Monitoring; } \\
\text { Olea europaea; Olive harvester }\end{array}$ \\
\hline $\begin{array}{l}\text { Wang Z., Hu M., Zhai } \\
\text { G. }\end{array}$ & 2018 & $\begin{array}{l}\text { Sensors } \\
\text { (Switzerland) }\end{array}$ & $\begin{array}{l}\text { Convolutional neural networks; } \\
\text { Fruit quality detection; } \\
\text { Hyperspectral transmittance } \\
\text { image; Internal mechanical } \\
\text { damage detection; Machine } \\
\text { learning }\end{array}$ \\
\hline $\begin{array}{l}\text { Kim C.S., Hwang } \\
\text { J.H., Jung J.T. }\end{array}$ & 2017 & $\begin{array}{l}\text { Information } \\
\text { (Japan) }\end{array}$ & $\begin{array}{l}\text { 3-point bending specimen; } \\
\text { Buckling; CFRP; Damage; } \\
\text { Restoration }\end{array}$ \\
\hline $\begin{array}{l}\text { Kabir G., Sadiq R., } \\
\text { Tesfamariam S. }\end{array}$ & 2016 & $\begin{array}{l}\text { Structure and } \\
\text { Infrastructure } \\
\text { Engineering }\end{array}$ & $\begin{array}{l}\text { Bayesian belief network } \\
\text { (BBN); Fault tree analysis } \\
\text { (FTA); Fuzzy set theory; } \\
\text { Linguistic variables; Oil and } \\
\text { gas pipelines; Safety } \\
\text { assessment; Uncertainty }\end{array}$ \\
\hline $\begin{array}{l}\text { Dorval A.D., } \\
\text { Meredieu C., Danjon } \\
\text { F. }\end{array}$ & 2016 & $\begin{array}{l}\text { Annals of } \\
\text { Botany }\end{array}$ & $\begin{array}{l}\text { 3D root architecture; } \\
\text { Acclimation; Biomechanics; } \\
\text { Flexural stiffness; Forest tree; }\end{array}$ \\
\hline
\end{tabular}




\begin{tabular}{|c|c|c|c|}
\hline & & & $\begin{array}{l}\text { Maximum tensile load; Pinus } \\
\text { pinaster; Soil depth; Toppling; } \\
\text { Tree anchorage; Windthrow }\end{array}$ \\
\hline $\begin{array}{l}\text { Favillier A., Lopez- } \\
\text { Saez J., Corona C., } \\
\text { Trappmann D., Toe } \\
\text { D., Stoffel M., Rovéra } \\
\text { G., Berger F. }\end{array}$ & 2015 & Geomorphology & $\begin{array}{l}\text { Coppice stands; } \\
\text { Dendrogeomorphology; Forest- } \\
\text { rockfall interactions; French } \\
\text { Alps; Recurrence intervals; } \\
\text { Submontane broadleaved } \\
\text { species }\end{array}$ \\
\hline $\begin{array}{l}\text { Alves V., Cury A., } \\
\text { Roitman N., Magluta } \\
\text { C., Cremona C. }\end{array}$ & 2015 & $\begin{array}{l}\text { Engineering } \\
\text { Structures }\end{array}$ & $\begin{array}{l}\text { Damage assessment; Learning } \\
\text { algorithms; Pattern recognition; } \\
\text { SHM; Symbolic data }\end{array}$ \\
\hline
\end{tabular}

(Alves et al., 2015; Andrejiova, Grincova, \& Marasova, 2019; Bhowmik, Krishnan, Hazra, \& Pakrashi, 2019; Dia, Dieng, Gaillet, \& Gning, 2019; Dorval, Meredieu, \& Danjon, 2016; Egnew, Roueche, \& Prevatt, 2018; Favillier et al., 2015; Kabir, Sadiq, \& Tesfamariam, 2016; Kim, Hwang, \& Jung, 2017; Noori Hoshyar, Samali, Liyanapathirana, \& Taghavipour, 2019; Pérez-Ruiz et al., 2018; Wang et al., 2018; Z. Zhang et al., 2019)

Table. 4 Notable ensembles models including it includes boosting and bagging for making ensembles

\begin{tabular}{|l|l|l|l|}
\hline Authors & Title & Year & Author Keywords \\
\hline $\begin{array}{l}\text { Mayer A.E., Mayer } \\
\text { P.N. }\end{array}$ & $\begin{array}{l}\text { Evolution of pore ensemble in solid and } \\
\text { molten aluminum under dynamic tensile } \\
\text { fracture: Molecular dynamics simulations } \\
\text { and mechanical models }\end{array}$ & 2019 & $\begin{array}{l}\text { Aluminum melt; Dynamic tensile } \\
\text { fracture; High-rate stretching; } \\
\text { Mechanical model; Molecular } \\
\text { dynamics; Solid aluminum }\end{array}$ \\
\hline $\begin{array}{l}\text { Uzay C., Geren N., } \\
\text { Boztepe M.H., } \\
\text { Bayramoglu M. }\end{array}$ & $\begin{array}{l}\text { Bending behavior of sandwich structures } \\
\text { with different fiber facing types and } \\
\text { extremely low-density foam cores }\end{array}$ & 2019 & $\begin{array}{l}\text { ANOVA; Failure modes; Flexural } \\
\text { properties; Sandwich structures; Ttree- } \\
\text { point bending }\end{array}$ \\
\hline $\begin{array}{l}\text { Panettieri E., } \\
\text { Guitard J. }\end{array}$ & $\begin{array}{l}\text { Mixed-mode crack propagation tests of } \\
\text { composite bonded joints using a dual- } \\
\text { actuator load frame - Constant and } \\
\text { variable G II /G I conditions }\end{array}$ & $\begin{array}{l}\text { Application of deep learning } \\
\text { architectures for accurate and rapid } \\
\text { detection of internal mechanical damage } \\
\text { of blueberry using hyperspectral } \\
\text { transmittance data }\end{array}$ & $\begin{array}{l}\text { Composite bonded joints; Debonding; } \\
\text { Dual-actuator system; Fracture } \\
\text { toughness; Mixed-mode }\end{array}$ \\
\hline $\begin{array}{l}\text { Detection and assessment of flaws in } \\
\text { friction stir welded joints using ultrasonic }\end{array}$ & 2018 & $\begin{array}{l}\text { Convolutional neural networks; Fruit } \\
\text { quality detection; Hyperspectral } \\
\text { transmittance image; Internal } \\
\text { mechanical damage detection; }\end{array}$ \\
\hline $\begin{array}{l}\text { Fakih M.A., Hu M., } \\
\text { Mustapha S., Tarraf }\end{array}$ & $\begin{array}{l}\text { Machine learning } \\
\text { CT scanning; Finite element analysis; } \\
\text { Friction stir welding; Lamb waves; }\end{array}$ \\
\hline
\end{tabular}




\begin{tabular}{|c|c|c|c|}
\hline $\begin{array}{l}\text { J., Ayoub G., } \\
\text { Hamade R. }\end{array}$ & $\begin{array}{l}\text { guided waves: experimental and finite } \\
\text { element analysis }\end{array}$ & & $\begin{array}{l}\text { Structural health monitoring; Weld } \\
\text { inspection }\end{array}$ \\
\hline $\begin{array}{l}\text { Froustey C., } \\
\text { Naimark O.B., } \\
\text { Panteleev I.A., } \\
\text { Bilalov D.A., } \\
\text { Petrova A.N., } \\
\text { Lyapunova E.A. }\end{array}$ & $\begin{array}{l}\text { Multiscale structural relaxation and } \\
\text { adiabatic shear failure mechanisms }\end{array}$ & 2017 & $\begin{array}{l}\text { adiabatic shear; dynamic loading; } \\
\text { microdefects }\end{array}$ \\
\hline $\begin{array}{l}\text { Tanırcan G., Alçık } \\
\text { H., Beyen K. }\end{array}$ & $\begin{array}{l}\text { Reliability of MEMS accelerometers for } \\
\text { instrumental intensity mapping of } \\
\text { earthquakes }\end{array}$ & 2017 & \\
\hline Li J., Zhang J. & $\begin{array}{l}\text { Adaptive Multiscale Noise Control } \\
\text { Enhanced Stochastic Resonance Method } \\
\text { Based on Modified EEMD with Its } \\
\text { Application in Bearing Fault Diagnosis }\end{array}$ & 2016 & \\
\hline $\begin{array}{l}\text { Yu J.-B., Lu X.-L., } \\
\text { Zong W.-Z. }\end{array}$ & $\begin{array}{l}\text { Wafer defect detection and recognition } \\
\text { based on local and nonlocal linear } \\
\text { discriminant analysis and dynamic } \\
\text { ensemble of gaussian mixture models }\end{array}$ & 2016 & $\begin{array}{l}\text { Gaussian mixture model (GMM); } \\
\text { Manifold learning; Pattern } \\
\text { recognition; Semiconductor } \\
\text { manufacturing; Wafer defect }\end{array}$ \\
\hline $\begin{array}{l}\text { Sokovikov M., } \\
\text { Bilalov D., Oborin } \\
\text { V., Chudinov V., } \\
\text { Uvarov S., } \\
\text { Bayandin Y., } \\
\text { Naimark O. }\end{array}$ & $\begin{array}{l}\text { Structural mechanisms of formation of } \\
\text { adiabatic shear bands }\end{array}$ & 2016 & $\begin{array}{l}\text { Dynamic loading; Microdefects; } \\
\text { Numerical modeling; Plastic strain } \\
\text { localization }\end{array}$ \\
\hline Ren Y.-C., Weng P. & $\begin{array}{l}\text { Structural damage detection based on } \\
\text { improved Hilbert-Huang transform }\end{array}$ & 2015 & $\begin{array}{l}\text { Damage detection; Ensemble } \\
\text { empirical mode decomposition; } \\
\text { Hilbert-huang transform; } \\
\text { Instantaneous frequency }\end{array}$ \\
\hline $\begin{array}{l}\text { Ovid'Ko I., } \\
\text { Sheinerman A., } \\
\text { Skiba N., } \\
\text { Krasnitiskiy S., } \\
\text { Smirnov A. }\end{array}$ & $\begin{array}{l}\text { Twin boundary migration and nanocrack } \\
\text { generation in ultrafine- grained materials } \\
\text { with nanotwinned structure }\end{array}$ & 2015 & $\begin{array}{l}\text { Cracks; Defects; Fracture; Modeling; } \\
\text { Nanotwinned materials; Plastic } \\
\text { deformation; Yield strength }\end{array}$ \\
\hline
\end{tabular}

(Fakih, Mustapha, Tarraf, Ayoub, \& Hamade, 2018; Froustey et al., 2017; J. Li \& Zhang, 2016; Mayer \& Mayer, 2019; Ovid'Ko, Sheinerman, Skiba, Krasnitiskiy, \& Smirnov, 2015; Panettieri, Leclerc, Jumel, \& Guitard, 2018; Y. C. Ren \& Weng, 2015; Sokovikov et al., 2016; Tanırcan, Alçık, \& Beyen, 2017; Uzay, Geren, Boztepe, \& Bayramoglu, 2019; Wang et al., 2018; Yu, Lu, \& Zong, 2016) 
Table 5. Notable Bayesians Models

\begin{tabular}{|c|c|c|c|}
\hline Authors & Year & Author Keywords & Title \\
\hline $\begin{array}{l}\text { Sha G., } \\
\text { Radzieński M., } \\
\text { Cao M., } \\
\text { Ostachowicz W. }\end{array}$ & 2019 & $\begin{array}{l}\text { Bayesian data fusion; Damage growth } \\
\text { monitoring; Damage localization; Relative } \\
\text { natural frequency change; Severity estimation }\end{array}$ & $\begin{array}{l}\text { A novel method for } \\
\text { single and multiple } \\
\text { damage detection in } \\
\text { beams using relative } \\
\text { natural frequency } \\
\text { changes }\end{array}$ \\
\hline $\begin{array}{l}\text { Chattopadhyay } \\
\text { P., Mondal S., } \\
\text { Ray A., } \\
\text { Mukhopadhyay } \\
\text { A. }\end{array}$ & 2019 & & $\begin{array}{l}\text { Dynamic Data- } \\
\text { Driven Combustor } \\
\text { Design for } \\
\text { Mitigation of } \\
\text { Thermoacoustic } \\
\text { Instabilities }\end{array}$ \\
\hline $\begin{array}{l}\text { Yang D.Y., } \\
\text { Frangopol D.M. }\end{array}$ & 2018 & $\begin{array}{l}\text { Bayesian network; crack growth; decision- } \\
\text { making; Fatigue; influence diagram; life-cycle }\end{array}$ & $\begin{array}{l}\text { Evidence-based } \\
\text { framework for real- } \\
\text { time life-cycle } \\
\text { management of } \\
\text { fatigue-critical } \\
\text { details of structures }\end{array}$ \\
\hline $\begin{array}{l}\text { Ye D., Hong } \\
\text { G.S., Zhang Y., } \\
\text { Zhu K., Fuh } \\
\text { J.Y.H. }\end{array}$ & 2018 & $\begin{array}{l}\text { Additive manufacturing; Deep belief networks; } \\
\text { Defect detection; Fast Fourier transform }\end{array}$ & $\begin{array}{l}\text { Defect detection in } \\
\text { selective laser } \\
\text { melting technology } \\
\text { by acoustic signals } \\
\text { with deep belief } \\
\text { networks }\end{array}$ \\
\hline $\begin{array}{l}\text { Ebrahimian H., } \\
\text { Astroza R., } \\
\text { Conte J.P., } \\
\text { Papadimitriou C. }\end{array}$ & 2018 & $\begin{array}{l}\text { Bayesian method; direct differentiation method; } \\
\text { joint parameter and input estimation; nonlinear } \\
\text { finite element model; output-only system } \\
\text { identification; structural health monitoring }\end{array}$ & $\begin{array}{l}\text { Bayesian optimal } \\
\text { estimation for } \\
\text { output-only } \\
\text { nonlinear system } \\
\text { and damage } \\
\text { identification of civil } \\
\text { structures }\end{array}$ \\
\hline $\begin{array}{l}\text { Wu X., Zeng X., } \\
\text { Huang J., Song } \\
\text { H.-Q. }\end{array}$ & 2017 & $\begin{array}{l}\text { Bayesian network; Finite element analysis; } \\
\text { Reliability growth; Solid rocket motor; Structure } \\
\text { optimization }\end{array}$ & $\begin{array}{l}\text { Research on Tail } \\
\text { Structure } \\
\text { Optimization for } \\
\text { Solid Rocket Motor }\end{array}$ \\
\hline $\begin{array}{l}\text { Liu Y., Shuai Q., } \\
\text { Zhou S., Tang J. }\end{array}$ & 2017 & $\begin{array}{l}\text { Damage prognosis; finite element methods } \\
\text { (FEMs); hierarchical Bayesian model; Markov }\end{array}$ & $\begin{array}{l}\text { Prognosis of } \\
\text { Structural Damage } \\
\text { Growth Via }\end{array}$ \\
\hline
\end{tabular}


chain Monte Carlo (MCMC) method; structural Integration of health monitoring

Physical Model

Prediction and

Bayesian Estimation

\begin{tabular}{|c|c|c|}
\hline $\begin{array}{l}\text { Ni Y., Lu X., Lu } \\
\text { W. }\end{array}$ & 2017 & $\begin{array}{l}\text { Bayesian; High-rise building; Modal } \\
\text { identification; Shaking table test; Vibration test }\end{array}$ \\
\hline $\begin{array}{l}\text { Ebrahimian H., } \\
\text { Astroza R., } \\
\text { Conte J.P., de } \\
\text { Callafon R.A. }\end{array}$ & 2017 & $\begin{array}{l}\text { Bayesian inference; Gradient-based } \\
\text { optimization; Model updating; Nonlinear finite } \\
\text { element model; Nonlinear system identification; } \\
\text { Uncertainty quantification }\end{array}$ \\
\hline $\begin{array}{l}\text { Kabir G., Sadiq } \\
\text { R., Tesfamariam } \\
\text { S. }\end{array}$ & 2016 & $\begin{array}{l}\text { Bayesian belief network (BBN); Fault tree } \\
\text { analysis (FTA); Fuzzy set theory; Linguistic } \\
\text { variables; Oil and gas pipelines; Safety } \\
\text { assessment; Uncertainty }\end{array}$ \\
\hline
\end{tabular}

Operational modal analysis of a highrise multi-function building with dampers by a Bayesian approach

Nonlinear finite element model updating for damage identification of civil structures using batch Bayesian estimation A fuzzy Bayesian
belief network for
safety assessment of
oil and gas pipelines Stochastic fatigue crack growth analysis of metallic structures under

Yazdanipour M., PourgolMohammad M. 2016

Bayesian approach; Crack length distribution; Fatigue crack growth; Probabilistic modeling; multiple thermalmechanical stress levels

The effect of wall thickness distribution on mechanical reliability and strength in Seuba J., Deville S., Guizard C., Stevenson A.J. 2016

Ceramics; Mechanical properties; Mechanical unidirectional porous ceramics

\section{Structural} modification

Alves V., Cury A., Roitman N., Magluta C., Cremona $\mathrm{C}$.
Damage assessment; Learning algorithms; 2015 assessment using supervised learning methods applied to vibration data 
Baneen U., Guivant J.E.
Bayesian; Curvature mode shapes; Damage

2015
A 2D Bayesian approach for damage detection in platetype structures

(Chattopadhyay, Mondal, Ray, \& Mukhopadhyay, 2019; Ebrahimian, Astroza, Conte, \& de Callafon, 2017; Ebrahimian, Astroza, Conte, \& Papadimitriou, 2018; Kabir et al., 2016; Liu, Shuai, Zhou, \& Tang, 2017; Y. Ni, Lu, \& Lu, 2017; Seuba, Deville, Guizard, \& Stevenson, 2016; Sha, Radzieński, Cao, \& Ostachowicz, 2019; Wu, Zeng, Huang, \& Song, 2017; Yang \& Frangopol, 2018; Yazdanipour \& Pourgol-Mohammad, 2016; Ye, Hong, Zhang, Zhu, \& Fuh, 2018)

\section{Logistic regressions}

\section{Table 6. Notable logistic regressions}

\begin{tabular}{|c|c|c|c|}
\hline Authors & Title & Year & Author Keywords \\
\hline $\begin{array}{l}\text { Egnew A.C., } \\
\text { Roueche D.B., } \\
\text { Prevatt D.O. }\end{array}$ & $\begin{array}{l}\text { Linking Building Attributes and } \\
\text { Tornado Vulnerability Using a } \\
\text { Logistic Regression Model }\end{array}$ & 2018 & \\
\hline $\begin{array}{l}\text { Jang D.-W., Lee } \\
\text { S., Park J.-W., } \\
\text { Baek D.-C. }\end{array}$ & $\begin{array}{l}\text { Failure detection technique under } \\
\text { random fatigue loading by } \\
\text { machine learning and dual sensing } \\
\text { on symmetric structure }\end{array}$ & 2018 & $\begin{array}{l}\text { Dual sensing; Failure } \\
\text { detection; Fatigue loading; } \\
\text { Machine learning; } \\
\text { Prognostics and Health } \\
\text { Management (PHM) } \\
\end{array}$ \\
\hline $\begin{array}{l}\text { Bissacot } \\
\text { A.C.G., Salgado } \\
\text { S.A.B., } \\
\text { Balestrassi P.P., } \\
\text { Paiva A.P., } \\
\text { Zambroni Souza } \\
\text { A.C., Wazen R. }\end{array}$ & $\begin{array}{l}\text { Comparison of neural networks } \\
\text { and logistic regression in } \\
\text { assessing the occurrence of } \\
\text { failures in steel structures of } \\
\text { transmission lines }\end{array}$ & 2016 & $\begin{array}{l}\text { Artificial neural networks; } \\
\text { Fall of metal structures; } \\
\text { Logistic regression; ROC } \\
\text { curves; Transmission lines }\end{array}$ \\
\hline $\begin{array}{l}\text { Regan T., } \\
\text { Canturk R., } \\
\text { Slavkovsky E., } \\
\text { Niezrecki C., } \\
\text { Inalpolat M. }\end{array}$ & $\begin{array}{l}\text { Wind turbine blade damage } \\
\text { detection using various machine } \\
\text { learning algorithms }\end{array}$ & 2016 & $\begin{array}{l}\text { Health Monitoring; Logistic } \\
\text { Regression; Machine } \\
\text { Learning; Support Vector } \\
\text { Machine; Wind Turbine } \\
\text { Blades }\end{array}$ \\
\hline $\begin{array}{l}\text { Gauthier F., } \\
\text { Hétu B., Allard } \\
\text { M. }\end{array}$ & $\begin{array}{l}\text { Forecasting method of ice blocks } \\
\text { fall using logistic model and } \\
\text { melting degree-days calculation: }\end{array}$ & 2015 & $\begin{array}{l}\text { Degree-day; Ice avalanche; } \\
\text { Ice blocks fall; Logistic } \\
\text { regression; Predictive model }\end{array}$ \\
\hline
\end{tabular}


a case study in northern Gaspésie,

Québec, Canada

Zhang W., Shen

S., Basak P.,

Wen H., Wu S.,

Faheem A.,

Mohammad

Development of predictive

models for initiation and

L.N.

propagation of field transverse

cracking

2015

Wazen R.N.,

Fernandes

T.S.P., Aoki

Evaluation of the susceptibility of

A.R., De Souza

failures in steel structures of

W.E.

transmission lines

Dropped structures; Logistic regression; Metallic structures; Rough sets;

2013

Transmission lines

(Bissacot et al., 2016; Egnew et al., 2018; Gauthier, Hétu, \& Allard, 2015; Jang et al., 2018; Regan, Canturk, Slavkovsky, Niezrecki, \& Inalpolat, 2016; Wazen, Fernandes, Aoki, \& De Souza, 2013; W. Zhang et al., 2015)

\section{Neuro-Fuzzy}

Table 7. Notable ANFIS models

\begin{tabular}{|c|c|c|c|}
\hline Authors & Title & Year & Author Keywords \\
\hline $\begin{array}{l}\text { Tran Q.T., } \\
\text { Nguyen S.D., } \\
\text { Seo T.-I. }\end{array}$ & $\begin{array}{l}\text { Algorithm for estimating } \\
\text { online bearing fault upon } \\
\text { the ability to extract } \\
\text { meaningful information } \\
\text { from big data of intelligent } \\
\text { structures }\end{array}$ & 2019 & $\begin{array}{l}\text { adaptive neuro-fuzzy inference system } \\
\text { (ANFIS)-based damage identification; AI } \\
\text { for estimating damage; identifying bearing } \\
\text { damage; singular spectrum analysis (SSA) } \\
\text { for identifying damage }\end{array}$ \\
\hline $\begin{array}{l}\text { Naderpour } \\
\text { H., Mirrashid } \\
\text { M. }\end{array}$ & $\begin{array}{l}\text { Shear failure capacity } \\
\text { prediction of concrete } \\
\text { beam-column joints in } \\
\text { terms of ANFIS and } \\
\text { GMDH }\end{array}$ & 2019 & $\begin{array}{l}\text { Adaptive neuro-fuzzy inference system } \\
\text { (ANFIS); Concrete beam-column joint; } \\
\text { Group method of data handling (GMDH); } \\
\text { Shear capacity; Soft computing; } \\
\text { Vulnerability }\end{array}$ \\
\hline $\begin{array}{l}\text { Hashemineja } \\
\text { d M.M., } \\
\text { Sohankar N., } \\
\text { Hajiannia A. }\end{array}$ & $\begin{array}{l}\text { Predicting the collapsibility } \\
\text { potential of unsaturated } \\
\text { soils using adaptive neural } \\
\text { fuzzy inference system and } \\
\text { particle swarm optimization }\end{array}$ & 2018 & $\begin{array}{l}\text { Adaptive neural fuzzy inference system; } \\
\text { Collapsibility potential; Gaussian } \\
\text { membership function; Particle swarm } \\
\text { optimization; Soft computing }\end{array}$ \\
\hline $\begin{array}{l}\text { Aydin K., } \\
\text { Kisi O. }\end{array}$ & $\begin{array}{l}\text { Damage detection in } \\
\text { structural beam elements }\end{array}$ & 2015 & $\begin{array}{l}\text { Beam; Damage detection; Grid } \\
\text { partitioning; Neuro fuzzy system; } \\
\text { Subtractive clustering }\end{array}$ \\
\hline
\end{tabular}




\begin{tabular}{|c|c|c|c|}
\hline & $\begin{array}{l}\text { using hybrid neuro fuzzy } \\
\text { systems }\end{array}$ & & \\
\hline $\begin{array}{l}\text { Guruprasad } \\
\text { R., Behera } \\
\text { B.K. }\end{array}$ & $\begin{array}{l}\text { Comparative Analysis of } \\
\text { Soft Computing Models in } \\
\text { Prediction of Bending } \\
\text { Rigidity of Cotton Woven } \\
\text { Fabrics }\end{array}$ & 2015 & $\begin{array}{l}\text { ANFIS; ANN; Bending rigidity; BPNN; } \\
\text { GANN }\end{array}$ \\
\hline $\begin{array}{l}\text { Nanda J., Das } \\
\text { L.D., Das S., } \\
\text { Das H.C. }\end{array}$ & $\begin{array}{l}\text { Influence of multi- } \\
\text { transverse crack on } \\
\text { cantilever shaft }\end{array}$ & 2015 & $\begin{array}{l}\text { adaptive neuro-fuzzy inference system; } \\
\text { experimental analysis; mode shape; } \\
\text { multiple cracks; natural frequency; shaft; } \\
\text { Vibration }\end{array}$ \\
\hline $\begin{array}{l}\text { [No author } \\
\text { name } \\
\text { available }]\end{array}$ & $\begin{array}{l}\text { 3rd International } \\
\text { Conference on Civil } \\
\text { Engineering and } \\
\text { Transportation, ICCET } \\
2013 \\
\end{array}$ & 2014 & \\
\hline $\begin{array}{l}\text { [No author } \\
\text { name } \\
\text { available] }\end{array}$ & $\begin{array}{l}2013 \text { International } \\
\text { Conference on Mechanical } \\
\text { and Electronics } \\
\text { Engineering, ICMEE } 2013\end{array}$ & 2013 & \\
\hline $\begin{array}{l}\text { [No author } \\
\text { name } \\
\text { available] }\end{array}$ & $\begin{array}{l}2013 \text { 2nd International } \\
\text { Conference on Manufacture } \\
\text { Engineering, Quality and } \\
\text { Production System, } \\
\text { ICMEQP } 2013\end{array}$ & 2013 & \\
\hline $\begin{array}{l}\text { Adoko A.C., } \\
\text { Gokceoglu } \\
\text { C., Wu L., } \\
\text { Zuo Q.J. }\end{array}$ & $\begin{array}{l}\text { Knowledge-based and data- } \\
\text { driven fuzzy modeling for } \\
\text { rockburst prediction }\end{array}$ & 2013 & $\begin{array}{l}\text { ANFIS; Mamdani fuzzy inference system; } \\
\text { Prediction modeling in rock engineering; } \\
\text { Rockburst; Takagi-Sugeno fuzzy inference } \\
\text { system }\end{array}$ \\
\hline
\end{tabular}

(Adoko, Gokceoglu, Wu, \& Zuo, 2013; Aydin \& Kisi, 2015; Guruprasad \& Behera, 2015; Hasheminejad, Sohankar, \& Hajiannia, 2018; Naderpour \& Mirrashid, 2019; Nanda, Das, Das, \& Das, 2015; Tran, Nguyen, \& Seo, 2019)

\section{Conclusions}

Deep learning methods are fast-evolving. Some of them have advanced to be specialized in a particular application domain. However, there is a gap in research in introducing the deep learning methods and summarize the methods and application in a single paper. Consequently, this paper aims at providing a comprehensive list of the most popular deep learning methods and provide notable applications. CNN, RNN, DAE, DBNs, LSTM 
methods have been identified as the most popular deep learning method. The description of each deep learning method and the function of each building block of them is explained.

\section{References}

Adoko, A. C., Gokceoglu, C., Wu, L., \& Zuo, Q. J. (2013). Knowledge-based and datadriven fuzzy modeling for rockburst prediction. International Journal of Rock Mechanics and Mining Sciences, 61, 86-95. doi:10.1016/j.ijrmms.2013.02.010

Alves, V., Cury, A., Roitman, N., Magluta, C., \& Cremona, C. (2015). Structural modification assessment using supervised learning methods applied to vibration data. Engineering Structures, 99, 439-448. doi:10.1016/j.engstruct.2015.05.003

AminShokravi, A., Eskandar, H., Derakhsh, A. M., Rad, H. N., \& Ghanadi, A. (2018). The potential application of particle swarm optimization algorithm for forecasting the airoverpressure induced by mine blasting. Engineering with Computers, 34(2), 277-285. doi:10.1007/s00366-017-0539-5

Andrejiova, M., Grincova, A., \& Marasova, D. (2019). Failure analysis of the rubber-textile conveyor belts using classification models. Engineering Failure Analysis, 101, 407-417. doi:10.1016/j.engfailanal.2019.04.001

Aydin, K., \& Kisi, O. (2015). Damage detection in structural beam elements using hybrid neuro fuzzy systems. Smart Structures and Systems, 16(6), 1107-1132. doi:10.12989/sss.2015.16.6.1107

Bhowmik, B., Krishnan, M., Hazra, B., \& Pakrashi, V. (2019). Real-time unified singleand multi-channel structural damage detection using recursive singular spectrum analysis. Structural Health Monitoring, 18(2), 563-589. doi:10.1177/1475921718760483

Bissacot, A. C. G., Salgado, S. A. B., Balestrassi, P. P., Paiva, A. P., Zambroni Souza, A. C., \& Wazen, R. (2016). Comparison of neural networks and logistic regression in assessing the occurrence of failures in steel structures of transmission lines. Open Electrical and Electronic Engineering Journal, 10, 11-26. doi:10.2174/1874129001610010011

Çalık, A., Yıldırım, S., \& Tosun, E. (2017). Estimation of crack propagation in polymer electrolyte membrane fuel cell under vibration conditions. International Journal of Hydrogen Energy, 42(36), 23347-23351. doi:10.1016/j.ijhydene.2017.02.119

Cha, Y. J., Choi, W., Suh, G., Mahmoudkhani, S., \& Büyüköztürk, O. (2018). Autonomous Structural Visual Inspection Using Region-Based Deep Learning for Detecting Multiple Damage Types. Computer-Aided Civil and Infrastructure Engineering, 33(9), 731-747. doi:10.1111/mice.12334 
Chattopadhyay, P., Mondal, S., Ray, A., \& Mukhopadhyay, A. (2019). Dynamic DataDriven Combustor Design for Mitigation of Thermoacoustic Instabilities. Journal of Dynamic Systems, Measurement and Control, Transactions of the ASME, 141(1). doi:10.1115/1.4040210

Chen, G., Li, T., Chen, Q., Ren, S., Wang, C., \& Li, S. (2019). Application of deep learning neural network to identify collision load conditions based on permanent plastic deformation of shell structures. Computational Mechanics, 64(2), 435-449. doi:10.1007/s00466-019-01706-2

Choi, C. K., Kim, J. S., \& Yoo, H. H. (2016). Identification of location and size of a defect in a structural system employing active external excitation and hybrid feature vector components in HMM. Journal of Mechanical Science and Technology, 30(6), 24272433. doi:10.1007/s12206-016-0502-1

Dia, A., Dieng, L., Gaillet, L., \& Gning, P. B. (2019). Damage detection of a hybrid composite laminate aluminum/glass under quasi-static and fatigue loadings by acoustic emission technique. Heliyon, 5(3). doi:10.1016/j.heliyon.2019.e01414

Dorval, A. D., Meredieu, C., \& Danjon, F. (2016). Anchorage failure of young trees in sandy soils is prevented by a rigid central part of the root system with various designs. Annals of Botany, 118(4), 747-762. doi:10.1093/aob/mcw098

Ebrahimian, H., Astroza, R., Conte, J. P., \& de Callafon, R. A. (2017). Nonlinear finite element model updating for damage identification of civil structures using batch Bayesian estimation. Mechanical Systems and Signal Processing, 84, 194-222. doi:10.1016/j.ymssp.2016.02.002

Ebrahimian, H., Astroza, R., Conte, J. P., \& Papadimitriou, C. (2018). Bayesian optimal estimation for output-only nonlinear system and damage identification of civil structures. Structural Control and Health Monitoring, 25(4). doi:10.1002/stc.2128

Egnew, A. C., Roueche, D. B., \& Prevatt, D. O. (2018). Linking Building Attributes and Tornado Vulnerability Using a Logistic Regression Model. Natural Hazards Review, 19(4). doi:10.1061/(ASCE)NH.1527-6996.0000305

Fakih, M. A., Mustapha, S., Tarraf, J., Ayoub, G., \& Hamade, R. (2018). Detection and assessment of flaws in friction stir welded joints using ultrasonic guided waves: experimental and finite element analysis. Mechanical Systems and Signal Processing, 101, 516-534. doi:10.1016/j.ymssp.2017.09.003

Favillier, A., Lopez-Saez, J., Corona, C., Trappmann, D., Toe, D., Stoffel, M., . . . Berger, F. (2015). Potential of two submontane broadleaved species (Acer opalus, Quercus pubescens) to reveal spatiotemporal patterns of rockfall activity. Geomorphology, 246, 35-47. doi:10.1016/j.geomorph.2015.06.010

Finotti, R. P., Cury, A. A., \& Barbosa, F. S. (2019). An SHM approach using machine learning and statistical indicators extracted from raw dynamic measurements. Latin American Journal of Solids and Structures, 16(2). doi:10.1590/1679-78254942 
Forero-Ramírez, J. C., Restrepo-Girón, A. D., \& Nope-Rodríguez, S. E. (2019). Detection of Internal Defects in Carbon Fiber Reinforced Plastic Slabs Using Background Thermal Compensation by Filtering and Support Vector Machines. Journal of Nondestructive Evaluation, 38(1). doi:10.1007/s10921-019-0569-6

Froustey, C., Naimark, O. B., Panteleev, I. A., Bilalov, D. A., Petrova, A. N., \& Lyapunova, E. A. (2017). Multiscale structural relaxation and adiabatic shear failure mechanisms. Physical Mesomechanics, 20(1), 31-42. doi:10.1134/S1029959917010039

Gauthier, F., Hétu, B., \& Allard, M. (2015). Forecasting method of ice blocks fall using logistic model and melting degree-days calculation: a case study in northern Gaspésie, Québec, Canada. Natural Hazards, 79(2), 855-880. doi:10.1007/s11069-015-1880-x

Gedik, N. (2018). Least squares support vector mechanics to predict the stability number of rubble-mound breakwaters. Water (Switzerland), 10(10). doi:10.3390/w10101452

Ghritlahre, H. K., \& Prasad, R. K. (2018). Exergetic performance prediction of a roughened solar air heater using artificial neural network. Journal of Mechanical Engineering, 64(3), 195-206. doi:10.5545/sv-jme.2017.4575

Gomes, G. F., de Almeida, F. A., Junqueira, D. M., da Cunha, S. S., Jr., \& Ancelotti, A. C., Jr. (2019). Optimized damage identification in CFRP plates by reduced mode shapes and GA-ANN methods. Engineering Structures, 181, 111-123. doi:10.1016/j.engstruct.2018.11.081

Gordan, M., Razak, H. A., Ismail, Z., \& Ghaedi, K. (2017). Recent developments in damage identification of structures using data mining. Latin American Journal of Solids and Structures, 14(13), 2373-2401. doi:10.1590/1679-78254378

Gordan, M., Razak, H. A., Ismail, Z., \& Ghaedi, K. (2018). Data mining based damage identification using imperialist competitive algorithm and artificial neural network. Latin American Journal of Solids and Structures, 15(8). doi:10.1590/1679-78254546

Güneyisi, E. M., Mermerdaş, K., Güneyisi, E., \& Gesoğlu, M. (2015). Numerical modeling of time to corrosion induced cover cracking in reinforced concrete using soft-computing based methods. Materials and Structures/Materiaux et Constructions, 48(6), 17391756. doi:10.1617/s11527-014-0269-8

Guruprasad, R., \& Behera, B. K. (2015). Comparative Analysis of Soft Computing Models in Prediction of Bending Rigidity of Cotton Woven Fabrics. Journal of The Institution of Engineers (India): Series E, 96(2), 167-174. doi:10.1007/s40034-014-0047-8

Harish, N., Mandal, S., Rao, S., \& Patil, S. G. (2015). Particle Swarm Optimization based support vector machine for damage level prediction of non-reshaped berm breakwater. Applied Soft Computing Journal, 27, 313-321. doi:10.1016/j.asoc.2014.10.041

Hasheminejad, M. M., Sohankar, N., \& Hajiannia, A. (2018). Predicting the collapsibility potential of unsaturated soils using adaptive neural fuzzy inference system and particle swarm optimization. Scientia Iranica, 25(6A), 2980-2996. doi:10.24200/sci.2018.20176 
Hong, S., Nomura, K. I., Krishnamoorthy, A., Rajak, P., Sheng, C., Kalia, R. K., . . . Vashishta, P. (2019). Defect Healing in Layered Materials: A Machine LearningAssisted Characterization of MoS2 Crystal Phases. Journal of Physical Chemistry Letters, 10(11), 2739-2744. doi:10.1021/acs.jpclett.9b00425

Huang, C., \& Wang, Z. (2018). Simplified model for estimating the punching load and deformation of RC flat plate based on big data mining. Journal of Intelligent and Fuzzy Systems, 35(1), 445-459. doi:10.3233/JIFS-169602

Jafari-Marandi, R., Khanzadeh, M., Tian, W., Smith, B., \& Bian, L. (2019). From in-situ monitoring toward high-throughput process control: cost-driven decision-making framework for laser-based additive manufacturing. Journal of Manufacturing Systems, 51, 29-41. doi:10.1016/j.jmsy.2019.02.005

Jang, D. W., Lee, S., Park, J. W., \& Baek, D. C. (2018). Failure detection technique under random fatigue loading by machine learning and dual sensing on symmetric structure. International Journal of Fatigue, 114, 57-64. doi:10.1016/j.ijfatigue.2018.05.004

Kabir, G., Sadiq, R., \& Tesfamariam, S. (2016). A fuzzy Bayesian belief network for safety assessment of oil and gas pipelines. Structure and Infrastructure Engineering, 12(8), 874-889. doi:10.1080/15732479.2015.1053093

Kan, C. D., Chen, W. L., Lin, C. H., Wang, J. N., Lu, P. J., Chan, M. Y., \& Wu, J. T. (2017). Handmade Trileaflet Valve Design and Validation for Pulmonary Valved Conduit Reconstruction Using Taguchi Method and Cascade Correlation Machine Learning Model. IEEE Access, 6, 7088-7099. doi:10.1109/ACCESS.2017.2782686

Kim, C. S., Hwang, J. H., \& Jung, J. T. (2017). A study on the cost-effective reliability centered maintenance of running gear system for rolling stock. Information (Japan), 20(5), 3649-3656. Retrieved from https://www.scopus.com/inward/record.uri?eid=2s2.0-85035014723\&partnerID=40\&md5=3f2b2e640ace0ebfae306fec2a8db6f1

Krummenacher, G., Ong, C. S., Koller, S., Kobayashi, S., \& Buhmann, J. M. (2018). Wheel Defect Detection with Machine Learning. IEEE Transactions on Intelligent Transportation Systems, 19(4), 1176-1187. doi:10.1109/TITS.2017.2720721

Li, H., Kafka, O. L., Gao, J., Yu, C., Nie, Y., Zhang, L., . . Liu, W. K. (2019). Clustering discretization methods for generation of material performance databases in machine learning and design optimization. Computational Mechanics, 64(2), 281-305. doi:10.1007/s00466-019-01716-0

Li, J., \& Zhang, J. (2016). Adaptive Multiscale Noise Control Enhanced Stochastic Resonance Method Based on Modified EEMD with Its Application in Bearing Fault Diagnosis. Shock and Vibration, 2016. doi:10.1155/2016/1485412

Lin, T. K. (2018). An edge-feature-description-based scheme combined with support vector machines for the detection of vortex-induced vibration. International Journal of Innovative Computing, Information and Control, 14(3), 833-845. Retrieved from https://www.scopus.com/inward/record.uri?eid=2-s2.0$\underline{85046408628 \& \text { partnerID }=40 \& \text { md5 }=\text { deade9209f23a44101 } 195 \mathrm{de} 2789870 \mathrm{c}}$ 
Lin, Y. Z., Nie, Z. H., \& Ma, H. W. (2017). Structural Damage Detection with Automatic Feature-Extraction through Deep Learning. Computer-Aided Civil and Infrastructure Engineering, 32(12), 1025-1046. doi:10.1111/mice.12313

Liu, Y., Shuai, Q., Zhou, S., \& Tang, J. (2017). Prognosis of Structural Damage Growth Via Integration of Physical Model Prediction and Bayesian Estimation. IEEE Transactions on Reliability, 66(3), 700-711. doi:10.1109/TR.2017.2713760

Lu, S., Jiang, M., Sui, Q., Sai, Y., \& Jia, L. (2015). Damage identification system of CFRP using fiber bragg grating sensors. Composite Structures, 125, 400-406. doi:10.1016/j.compstruct.2015.02.038

Mayer, A. E., \& Mayer, P. N. (2019). Evolution of pore ensemble in solid and molten aluminum under dynamic tensile fracture: Molecular dynamics simulations and mechanical models. International Journal of Mechanical Sciences, 157-158, 816-832. doi:10.1016/j.ijmecsci.2019.05.023

Meruane, V., \& Ortiz-Bernardin, A. (2015). Structural damage assessment using linear approximation with maximum entropy and transmissibility data. Mechanical Systems and Signal Processing, 54, 210-223. doi:10.1016/j.ymssp.2014.08.018

Naderpour, H., \& Mirrashid, M. (2019). Shear failure capacity prediction of concrete beamcolumn joints in terms of ANFIS and GMDH. Practice Periodical on Structural Design and Construction, 24(2). doi:10.1061/(ASCE)SC.1943-5576.0000417

Nair, A., Cai, C. S., \& Kong, X. (2019a). Acoustic emission pattern recognition in CFRP retrofitted RC beams for failure mode identification. Composites Part B: Engineering, 161, 691-701. doi:10.1016/j.compositesb.2018.12.120

Nair, A., Cai, C. S., \& Kong, X. (2019b). Studying Failure Modes of GFRP Laminate Coupons Using AE Pattern-Recognition Method. Journal of Aerospace Engineering, 32(4). doi:10.1061/(ASCE)AS.1943-5525.0001015

Nanda, J., Das, L. D., Das, S., \& Das, H. C. (2015). Influence of multi-transverse crack on cantilever shaft. International Journal of Damage Mechanics, 24(7), 1047-1069. doi:10.1177/1056789514560916

Ni, F., Zhang, J., \& Chen, Z. (2019). Zernike-moment measurement of thin-crack width in images enabled by dual-scale deep learning. Computer-Aided Civil and Infrastructure Engineering, 34(5), 367-384. doi:10.1111/mice.12421

Ni, Y., Lu, X., \& Lu, W. (2017). Operational modal analysis of a high-rise multi-function building with dampers by a Bayesian approach. Mechanical Systems and Signal Processing, 86, 286-307. doi:10.1016/j.ymssp.2016.10.009

Nie, W., Zhao, Z. Y., Goh, A. T. C., Song, M. K., Guo, W., \& Zhu, X. (2018). Performance based support design for horseshoe-shaped rock caverns using 2D numerical analysis. Engineering Geology, 245, 266-279. doi:10.1016/j.enggeo.2018.09.007

Noori Hoshyar, A., Samali, B., Liyanapathirana, R., \& Taghavipour, S. (2019). Analysis of failure in concrete and reinforced-concrete beams for the smart aggregate-based monitoring system. Structural Health Monitoring. doi:10.1177/1475921719854151 
Ovid'Ko, I., Sheinerman, A., Skiba, N., Krasnitiskiy, S., \& Smirnov, A. (2015). Twin boundary migration and nanocrack generation in ultrafine- grained materials with nanotwinned structure. WSEAS Transactions on Applied and Theoretical Mechanics, 10, 204-212. Retrieved from https://www.scopus.com/inward/record.uri?eid=2-s2.0-

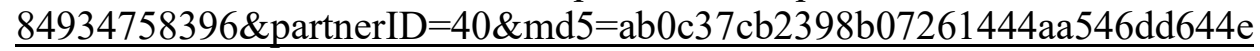

Panettieri, E., Leclerc, G., Jumel, J., \& Guitard, J. (2018). Mixed-mode crack propagation tests of composite bonded joints using a dual-actuator load frame - Constant and variable G II /G I conditions. Engineering Fracture Mechanics, 202, 471-486. doi:10.1016/j.engfracmech.2018.09.015

Patala, S. (2019). Understanding grain boundaries - The role of crystallography, structural descriptors and machine learning. Computational Materials Science, 162, 281-294. doi:10.1016/j.commatsci.2019.02.047

Pérez-Ruiz, M., Rallo, P., Jiménez, M. R., Garrido-Izard, M., Suárez, M. P., Casanova, L., ... Morales-Sillero, A. (2018). Evaluation of over-the-row harvester damage in a superhigh-density olive orchard using on-board sensing techniques. Sensors (Switzerland), 18(4). doi:10.3390/s18041242

Pu, Y., Apel, D. B., Liu, V., \& Mitri, H. (2019). Machine learning methods for rockburst prediction-state-of-the-art review. International Journal of Mining Science and Technology. doi:10.1016/j.ijmst.2019.06.009

Quaranta, G., Lopez, E., Abisset-Chavanne, E., Duval, J. L., Huerta, A., \& Chinesta, F. (2019). Structural health monitoring by combining machine learning and dimensionality reduction techniques. Revista Internacional de Metodos Numericos para Calculo y Diseno en Ingenieria, 35(1). doi:10.23967/j.rimni.2018.12.001

Regan, T., Canturk, R., Slavkovsky, E., Niezrecki, C., \& Inalpolat, M. (2016). Wind turbine blade damage detection using various machine learning algorithms.

Ren, S., Chen, G., Li, T., Chen, Q., \& Li, S. (2018). A deep learning-based computational algorithm for identifying damage load condition: An artificial intelligence inverse problem solution for failure analysis. CMES - Computer Modeling in Engineering and Sciences, 117(3), 287-307. doi:10.31614/cmes.2018.04697

Ren, Y. C., \& Weng, P. (2015). Structural damage detection based on improved HilbertHuang transform. Zhendong yu Chongji/Journal of Vibration and Shock, 34(18), 195199. doi:10.13465/j.cnki.jvs.2015.18.033

Rezaniaiee Aqdam, H., Ettefagh, M. M., \& Hassannejad, R. (2018). Health monitoring of mooring lines in floating structures using artificial neural networks. Ocean Engineering, 164, 284-297. doi:10.1016/j.oceaneng.2018.06.056

Rojas-Moraleda, R., Valous, N. A., Gowen, A., Esquerre, C., Härtel, S., Salinas, L., \& O'Donnell, C. (2017). A frame-based ANN for classification of hyperspectral images: assessment of mechanical damage in mushrooms. Neural Computing and Applications, 28, 969-981. doi:10.1007/s00521-016-2376-7 
Salehi, H., Das, S., Biswas, S., \& Burgueño, R. (2019). Data mining methodology employing artificial intelligence and a probabilistic approach for energy-efficient structural health monitoring with noisy and delayed signals. Expert Systems with Applications, 135, 259-272. doi:10.1016/j.eswa.2019.05.051

Samareh, H., Khoshrou, S. H., Shahriar, K., Ebadzadeh, M. M., \& Eslami, M. (2017). Optimization of a nonlinear model for predicting the ground vibration using the combinational particle swarm optimization-genetic algorithm. Journal of African Earth Sciences, 133, 36-45. doi:10.1016/j.jafrearsci.2017.04.029

Seuba, J., Deville, S., Guizard, C., \& Stevenson, A. J. (2016). The effect of wall thickness distribution on mechanical reliability and strength in unidirectional porous ceramics. Science and Technology of Advanced Materials, 17(1), 128-135. doi:10.1080/14686996.2016.1140309

Sha, G., Radzieński, M., Cao, M., \& Ostachowicz, W. (2019). A novel method for single and multiple damage detection in beams using relative natural frequency changes. Mechanical Systems and Signal Processing, 132, 335-352. doi:10.1016/j.ymssp.2019.06.027

Sokovikov, M., Bilalov, D., Oborin, V., Chudinov, V., Uvarov, S., Bayandin, Y., \& Naimark, O. (2016). Structural mechanisms of formation of adiabatic shear bands. Frattura ed Integrita Strutturale, 10(38), 296-304. doi:10.3221/IGF-ESIS.38.40

Tan, Z. X., Thambiratnam, D. P., Chan, T. H. T., \& Abdul Razak, H. (2017). Detecting damage in steel beams using modal strain energy based damage index and Artificial Neural Network. Engineering Failure Analysis, 79, 253-262. doi:10.1016/j.engfailanal.2017.04.035

Tanırcan, G., Alçık, H., \& Beyen, K. (2017). Reliability of MEMS accelerometers for instrumental intensity mapping of earthquakes. Annals of Geophysics, 60(6). doi:10.4401/ag-7501

Tran, Q. T., Nguyen, S. D., \& Seo, T. I. (2019). Algorithm for estimating online bearing fault upon the ability to extract meaningful information from big data of intelligent structures. IEEE Transactions on Industrial Electronics, 66(5), 3804-3813. doi:10.1109/TIE.2018.2847704

Uzay, C., Geren, N., Boztepe, M. H., \& Bayramoglu, M. (2019). Bending behavior of sandwich structures with different fiber facing types and extremely low-density foam cores. Materialpruefung/Materials Testing, 61(3), 220-230. doi:10.3139/120.111311

Wang, Z., Hu, M., \& Zhai, G. (2018). Application of deep learning architectures for accurate and rapid detection of internal mechanical damage of blueberry using hyperspectral transmittance data. Sensors (Switzerland), 18(4). doi:10.3390/s18041126

Wazen, R. N., Fernandes, T. S. P., Aoki, A. R., \& De Souza, W. E. (2013). Evaluation of the susceptibility of failures in steel structures of transmission lines. Journal of Control, Automation and Electrical Systems, 24(1-2), 174-186. doi:10.1007/s40313-013-0019-0 
Wong, E. W. C., \& Kim, D. K. (2018). A simplified method to predict fatigue damage of TTR subjected to short-term VIV using artificial neural network. Advances in Engineering Software, 126, 100-109. doi:10.1016/j.advengsoft.2018.09.011

Wu, X., Zeng, X., Huang, J., \& Song, H. Q. (2017). Research on Tail Structure Optimization for Solid Rocket Motor. Tuijin Jishu/Journal of Propulsion Technology, 38(12), 28222829. doi:10.13675/j.cnki.tjjs.2017.12.022

Yang, D. Y., \& Frangopol, D. M. (2018). Evidence-based framework for real-time life-cycle management of fatigue-critical details of structures. Structure and Infrastructure Engineering, 14(5), 509-522. doi:10.1080/15732479.2017.1399150

Yazdanipour, M., \& Pourgol-Mohammad, M. (2016). Stochastic fatigue crack growth analysis of metallic structures under multiple thermal-mechanical stress levels. Materials and Design, 95, 599-611. doi:10.1016/j.matdes.2016.01.110

Ye, D., Hong, G. S., Zhang, Y., Zhu, K., \& Fuh, J. Y. H. (2018). Defect detection in selective laser melting technology by acoustic signals with deep belief networks. International Journal of Advanced Manufacturing Technology, 96(5-8), 2791-2801. doi:10.1007/s00170-018-1728-0

Yi, Q., Wang, H., Guo, R., Li, S., \& Jiang, Y. (2017). Laser ultrasonic quantitative recognition based on wavelet packet fusion algorithm and SVM. Optik, 149, 206-219. doi:10.1016/j.ijleo.2017.08.105

Yu, J. B., Lu, X. L., \& Zong, W. Z. (2016). Wafer defect detection and recognition based on local and nonlocal linear discriminant analysis and dynamic ensemble of gaussian mixture models. Zidonghua Xuebao/Acta Automatica Sinica, 42(1), 47-59. doi:10.16383/j.aas.2016.c150311

Zhang, W., Shen, S., Basak, P., Wen, H., Wu, S., Faheem, A., \& Mohammad, L. N. (2015) Development of predictive models for initiation and propagation of field transverse cracking. In: Vol. 2524. Transportation Research Record (pp. 92-99): National Research Council.

Zhang, Z., Hong, Y., Hou, B., Zhang, Z., Negahban, M., \& Zhang, J. (2019). Accelerated discoveries of mechanical properties of graphene using machine learning and highthroughput computation. Carbon, 148, 115-123. doi:10.1016/j.carbon.2019.03.046 